\title{
ESTUDANTES DE ENFERMAGEM RELATIVAMENTE À COMUNIDADE LGBT: CONHECIMENTOS, ATITUDES E COMPETÊNCIA CULTURAL
}

\author{
Nursing students regarding the LGBT community: knowledge, attitudes and cultural competence \\ Estudiantes de enfermería en relación con la comunidad LGBT: conocimientos, actitudes y competencia \\ cultural
}

\author{
Maribel Carvalhais, ${ }^{*}$ Andreia Santos, ${ }^{* *}$ Diana Portovedo, ${ }^{* * *}$ Marco Oliveira, ${ }^{* * * *}$ Rafaela \\ Barbosa, ${ }^{* * * * *}$ Sofia Costa, ${ }^{* * * * * *}$ Ana Torres, ${ }^{* * * * * * *}$
}

\begin{abstract}
Resumo
Enquadramento: as atitudes, conhecimentos e competência cultural dos estudantes de enfermagem relativamente à comunidade de Lésbicas, Gays, Bissexuais e Transgéneros (LGBT) tem repercussões no acesso e na qualidade dos cuidados de saúde destas minorias sexuais. Investigações demostram que, apesar do aumento de informação os estudantes de enfermagem, apresentam défices significativos nesta área. Objetivo: conhecer as variáveis associadas aos conhecimentos, atitudes e competência cultural dos estudantes de enfermagem portugueses relativos à comunidade LGBT. Metodologia: estudo descritivo-correlacional, com amostra de conveniência de uma instituição de Ensino Superior da região norte de Portugal. A recolha de dados foi realizada através de um questionário on-line, composto por questões sócio-demográficas, questões de verificação de conhecimentos e o Questionário Multidimensional do Preconceito Polimorfo (Lopes, Oliveira, Nogueira, \& Grave, 2016). Resultados: o estudo integrou uma amostra de 99 estudantes de licenciatura em enfermagem. Encontraram-se variáveis de risco para atitude discriminatória, como idade e ano académico, orientação heterossexual, residência em meios rurais, política de centro e falta de familiaridade com pessoas LGBT, bem como, associação com baixo conhecimento. Conclusão: podemos concluir que os estudantes de enfermagem demonstram necessitar de reforço de conhecimentos, para melhor adequação das atitudes e competências para lidar com a comunidade LGBT.
\end{abstract}

Palavras-chave: estudantes de enfermagem; conhecimento; atitude; minorias sexuais e de género

${ }^{*}$ PhD, Professora Adjunta na Escola Superior de Saúde Norte da Cruz Vermelha Portuguesa/CINTESIS

https://orcid.org/0000-0002-2206-2582

autor de correspondência: maribel.carvalhais@essnortecvp.pt

** RN, Enfermeira, Louromédica- Clínica Médica https://orcid.org/0000-0001-78302488

${ }^{* * *}$ RN, Enfermeira, Lar Nossa Senhora da Piedade https://orcid.org/0000-00027308-6373

${ }^{* * * *}$ RN, Enfermeiro, Centro Hospitalar Baixo Vouga, Serviço de Urgência https://orcid.org/0000-0003-2560-2457

${ }^{* * * * *} \mathrm{RN}, \quad$ Enfermeira, CentroDial https://orcid.org/0000-0001-6013-9993

${ }^{* * * * * *}$ RN, Enfermeira, Irmandade da Misericórdia de Albergaria-a-Velha https://orcid.org/0000-0002-2976-4667

*******PhD, Professora Adjunta na Escola Superior de Saúde Norte da Cruz Vermelha Portuguesa/CINTESIS

https://orcid.org/0000-0002-8919-0378

Como referenciar:

Carvalhais, M., Santos, A., Portovedo, D. Oliveira, M., Barbosa, R., Costa, S., \& Torres, A (2020). Estudantes de enfermagem relativamente à comunidade LGBT: conhecimentos, atitudes e competência cultural. Revista de Investigação \& Inovação em Saúde, vol.3(2). 61-73. doi.org/10.37914/riis.v3i2.95

Recebido para publicação em: 22/09/2020 Aceite para publicação em: 14/12/2020

\section{Abstract}

Background: the attitudes, knowledge, and cultural competence of nursing students towards the lesbian, gay, bisexual and transgender (LGBT) community have repercussions on the access and quality of healthcare of these sexual minorities. Research shows that, despite the increase in information, nursing students have significant deficits in this area. Objective: to know the variables related to the state of knowledge, attitudes and cultural competence of Portuguese nursing students in relation to the LGBT community. Methodology: descriptive-correlational study, with convenience sample from a Higher Education Institution in the north region of Portugal. Collection data was developed through an online questionnaire, composed of socialdemographic, knowledge verification questions and the Questionário Multidimensional do Preconceito Polimorfo (Lopes, Oliveira, Nogueira, \& Grave, 2016). Results: the study included a sample of 99 nursing undergraduate students. Risk variables for discriminatory attitude were found, such as age and academic year, heterosexual orientation, residence in rural areas, centre politics and lack of familiarity with LGBT people, as well as association with low knowledge. Conclusion: we can conclude that nursing students demonstrate the need for reinforcement of knowledge, to better adapt attitudes and skills to deal with the LGBT community. Keywords: nursing students; knowledge, attitude; sexual and gender minorities

\section{Resumen}

Marco contextual: las actitudes, conocimientos y competencias culturales de los estudiantes de salud en la comunidad de Lesbianas, Gays, Bisexuales y Transgénero (LGBT) tienen repercusiones en el acceso y en la calidad de los cuidados de la salud para estas minorías sexuales. Las investigaciones demuestran que, a pesar del aumento de la información, los estudiantes de enfermería presentan déficits significativos en este ámbito. Objetivo: conocer las variables asociadas a los conocimientos, actitudes y competencia cultural de los estudiantes de enfermería portugueses en relación a la comunidad LGBT. Metodología: estudio descriptivo-correlacional, con muestra de conveniencia de una institución de educación superior en la región norte de Portugal. La recogida de datos se realizó a través de un cuestionario en línea, compuesto por cuestiones sociales demográficos, cuestiones de verificación de conocimientos y el Questionário Multidimensional do Preconceito Polimorfo (Lopes, Oliveira, Nogueira, \& Grave, 2016). Resultados: el estudio integró 99 estudiantes del curso de enfermería. Se encontraron variables de riesgo para la actitud discriminatoria, como edad y año académico, orientación heterosexual, residencia en áreas rurales, política del centro y desconocimiento de personas LGBT, así como asociación con bajo conocimiento. Conclusión: estudiantes de enfermería demuestran la necesidad de refuerzo de conocimientos, con el fin de adaptar mejor las actitudes y habilidades para el trato con la comunidad LGBT.

Palabras clave: estudiantes de enfermería; conocimiento, actitude; minorías sexuales y de género 


\section{Estudantes de enfermagem relativamente à comunidade LGBT: conhecimentos, atitudes e competência cultural}

\section{INTRODUÇÃO}

Os estudantes de enfermagem vivenciam diversos desafios na vida académica, em parte colocados pela necessidade de adaptação às pessoas a quem têm de prestar cuidados, tendo em consideração as caraterísticas da personalidade de cada um, bem como, o seu contexto histórico, social, cultural e político.

A saúde das pessoas cuja orientação sexual as possas designar como pertencentes à comunidade de Lésbicas, Gays, Bissexuais e Transgéneros (LGBT), uma minoria populacional, apresenta constrangimentos, não só nas leis e práticas clínicas, como noutros contextos como a educação, o que faz com que, para melhorar a discrepância existente nos cuidados de saúde devido à identificação da orientação sexual de cada pessoa sejam necessários conhecimentos e competências culturais sobre este tema (Orgel, 2017; Lim \& Hsu, 2016).

$\mathrm{Na}$ literatura encontram-se identificados diferentes fatores dos estudantes de enfermagem que se mostram correlacionados e interferem de forma negativa nas atitudes, nos conhecimentos e na competência cultural relativamente à comunidade LGBT (Richardson, et al., 2016; Unlu, et al., 2016; Cornelius \& Carrick, 2015; Rowniak, 2015; Pinto, 2011). Existe consensual suporte científico que afirma a necessidade de criação de programas que potenciem o conhecimento dos estudantes de enfermagem sobre esta comunidade (Richardson, et al., 2016; Unlu, et al., 2016; Cornelius \& Carrick, 2015; Rowniak, 2015; Pinto, 2011).

Em suma, tendo em consideração a revisão da literatura científica realizada, e observando que é um assunto pouco explorado e gerador de comportamentos e atitudes pouco adequadas ou até discriminatórias, propomo-nos a conhecer as variáveis associadas aos conhecimentos, às atitudes e à competência cultural dos estudantes de enfermagem relativamente à comunidade de LGBT.

Neste âmbito, as 2 questões de investigação principais objetivadas a que se pretende dar resposta com a concretização deste estudo são: "Que variáveis apresentam associação às atitudes, conhecimentos e competência cultural dos estudantes de enfermagem portugueses perante pessoas LGBT?"; e "Que implicações pedagógicas, clínicas e comunitárias é que emergem dos resultados dos conhecimentos, atitudes e competência cultural dos estudantes de enfermagem portugueses relativamente à comunidade LGBT?".

\section{ENQUADRAMENTO/FUNDAMENTAÇÃO TEÓRICA}

$O$ contexto da comunidade LGBT exige a compreensão de conceitos que a permitem conhecer melhor. Em março de 2017, a ILGA Portugal atualizou a sua designação para "Intervenção Lésbica, Gay, Bissexual, Trans e Intersexo". Contextualiza-se que: 1) Lésbica (ou Lesbian) refere-se a uma mulher que sente atração emocional e/ou sexual por outras mulheres; 2) Gay refere-se a um homem que se sente atraído emocional e/ou sexualmente por outros homens; 3) Bisexual refere-se a uma pessoa que sente atração emocional e/ou sexual por pessoas de ambos os sexos; 4) Transgénero refere-se a uma pessoa que se identifica com um sexo diferente do seu sexo à nascença; 5) Transsexual refere-se a uma pessoa que está em processo ou já procedeu a tratamentos (que podem incluir cirurgia e tratamentos hormonais) para tornar o seu corpo congruente com o género sexual com que se identifica e 6) Intersexo refere-se às pessoas que 


\section{Estudantes de enfermagem relativamente à comunidade LGBT: conhecimentos, atitudes e competência cultural}

nasceram com caraterísticas sexuais (incluindo genitais, gónadas, padrões hormonais e/ou cromossomáticos) que não correspondem a noções binárias do corpo masculino ou feminino, descrevendo um largo espectro de variações corporais naturais (UNESCO, 2016).

Conforme é apresentado no Manual de Diagnóstico das Perturbações Mentais (American Psychiatric Association, 2014), a distinção entre os termos "sexo" e "género" é muito controversa e levou à proliferação de termos cujos significados variam ao longo do tempo e entre disciplinas. Neste manual é clarificado que "sexo" se refere a indicadores biológicos masculino/feminino; e "género" é usado para definir publicamente como rapaz e rapariga, homem ou mulher de acordo com os fatores sociais. Outro conceito distinto, por vezes utilizado indiscriminadamente de forma incorreta neste contexto é o de "transvestismo", designado como um fetichismo transvéstico, que consiste num tipo de parafilia (excitação sexual a estímulos nãoconvencionais) caracterizada pelo comportamento de vestir roupas do sexo oposto (Campbell, 2009). Uma das razões para a invisibilidade destes fenómenos na nossa sociedade, como noutras, decorre da sistemática confusão em termos de senso comum entre a transexualidade e/ou o transgénero e a homossexualidade. Trata-se de realidades diferentes. Na população portuguesa tem-se verificado mudanças legais no que diz respeito aos direitos da-população LGBT, de que damos alguns exemplos: uma significativa, a Lei no 9/2010 de 31 de Maio, que "Permite o casamento civil entre pessoas do mesmo sexo" (Assembleia da República, 2010, p. 1853); e a Lei no 38/2018 de 7 de agosto, que, no capítulo III
"Medidas de proteção" no Artigo 11 - "Saúde", refere que a pessoa que solicitar modificações ao nível do corpo e das caraterísticas sexuais tem o direito a serviços de referência ou unidades especializadas no Serviço Nacional de Saúde. Estas alterações legislativas dos últimos anos, refletem os avanços no reconhecimento da necessidade de adaptação das atitudes perante a comunidade LGBT, nomeadamente no contexto da saúde.

No que diz respeito às atitudes pode-se afirmar que consistem em tendências de reação relativamente a uma pessoa, objeto ou a uma situação do quotidiano e dependem do conhecimento cultural de cada indivíduo (Campbell, 2009). Assim, atendendo a este conceito, podemos verificar também que o conhecimento cultural enquadra a compreensão da existência de diversas crenças (religião), relações de género e de papéis sociais, impacto da classe socioeconómica, etnicidade, acessibilidade à educação, tradições, estrutura familiar, conceção de estado de saúde-doença, modos de comportamento, estilos de comunicação, impacto de reunião social e políticas de saúde (Rodrigues, Martins, \& Pereira, 2013). Posto isto, o termo "competência cultural" designa a capacidade de entender, aplicar e comunicar este conhecimento, relativa a uma cultura e raciocinar sobre riscos/benefícios de ações perante as diferenças culturais (Campbell, 2009).

A teoria transcultural de Leininger ajuda a uma melhor definição da competência cultural, pois refere que é o enfermeiro que implementa os cuidados de saúde ao paciente, estando a maior parte do tempo a seu lado, contendo assim um objetivo único em que o profissional de saúde tem o dever de apresentar educação cultural para que possa implementar os 


\section{Estudantes de enfermagem relativamente à comunidade LGBT: conhecimentos, atitudes e \\ competência cultural}

cuidados de saúde paralelamente ao estilo de cuidados que o paciente exige de acordo com as suas expectativas culturais $\mathrm{e}$ as suas necessidades (Betancourt, 2015). A educação cultural focada no conhecimento sobre a comunidade LGBT pode beneficiar não só os comportamentos dos profissionais de saúde no cuidado ao cliente, como também a efetividade em reduzir a discriminação, o preconceito e as atitudes negativas da população (Orgel, 2017).

Estes fenómenos sociais encontram-se relacionados com a heteronormatividade, sendo designados como um conjunto de crenças baseadas na ideia de dicotomia do género feminino e masculino (ILGA, 2019).

Verificam-se poucos estudos sobre atitudes, conhecimentos e competência cultural dos estudantes de enfermagem. Os estudos sobre esta temática retiram conclusões acerca de fatores que estão associados à discriminação da comunidade LGBT, afirmando os seguintes: falta de conhecimento e de ensino sobre a comunidade LGBT (Richardson, et al., 2016; Cornelius \& Carrick, 2015); religião (Richardson et al., 2016; Unlu, et al., 2016; Cornelius \& Carrick, 2015; Rowniak, 2015; Pinto, 2011) residência em meios rurais e orientação política de centro (Pinto, 2011); falta de familiaridade ou amizade com pessoas LGBT (Rowniak, 2015; Pinto, 2011; Unlu, et al., 2016); e serem estudantes mais velhos e do sexo masculino (Pinto, 2011; Unlu, et al., 2016). Vários estudos verificam que existem limitados conteúdos e estratégias educativas nos cursos de saúde para diminuir viéses de práticas clínicas com a comunidade LGBT, incentivando à investigação e à adoção de medidas que possam contribuir para o desenvolvimento desta área (Guzman, Moukoulou,
Scott, \& Zerwic, 2018; Maley \& Gross, 2019; Morris et al., 2019).

\section{METODOLOGIA}

Este trabalho consiste num estudo descritivocorrelacional. Foi utilizada uma amostra por conveniência de estudantes da licenciatura em enfermagem de uma instituição de ensino superior (IES) da região norte de Portugal. Foram excluídos os estudantes do programa Erasmus e/ou que possuem residência no estrangeiro uma vez que o objetivo deste estudo se foca apenas na população portuguesa/residente em Portugal, com vista a evitar o viés relacionado com variável parasita nos resultados obtidos.

Foram utilizados os seguintes instrumentos para recolha de dados, consistindo num questionário online que engloba questões sócio-demográficas, questões de verificação de conhecimentos e o Questionário Multidimensional do Preconceito Polimorfo (Lopes, Oliveira, Nogueira, \& Grave, 2016). O questionário sócio-demográfico recolheu dados como os seguintes: idade, género, orientação política, religião, entre outras, para avaliar fatores relacionados com diferentes atitudes perante a comunidade LGBT. Seguiram-se as questões de avaliação de conhecimentos sobre a comunidade LGBT, que se basearam em estudos anteriores (Davidson, 2014; Strong, 2013).

E, por último, foram colocadas questões do Questionário Multidimensional do Preconceito Polimorfo (Lopes, Oliveira, Nogueira, \& Grave, 2016), que é um questionário de carácter multidimensional composto por 6 subescalas (com escala de respostas de 1 = discordo totalmente a 5 = concordo totalmente) 


\section{Estudantes de enfermagem relativamente à comunidade LGBT: conhecimentos, atitudes e competência cultural}

que avaliam as atitudes da perante gays e lésbicas. Após análise integral das 6 subescalas decidimos pela utilização das 3 seguintes subescalas: F1 Heterossexismo tradicional $(a=.94)$, F4- Negação da discriminação continuada $(a=.73)$ e F5 - Valorização da afirmação gay $(a=.79)$. A primeira subescala (F1) avalia que o heterossexismo tradicional, ou seja, a crença da perceção das pessoas gays e lésbicas como pervertidas, pecadoras ou imorais às quais deveriam ser negados determinados direitos e privilégios. A segunda subescala (F4), avalia a negação da discriminação continuada, que diz respeito à tendência para a negação da contínua discriminação e às crenças de que a discriminação homofóbica não é um problema atual. A terceira subescala (F5) foca-se na avaliação da valorização da afirmação gay, e refere-se a crenças baseadas nos valores a favor da diversidade sexual. Quanto maior a pontuação obtida no total desta escala e respectivas subescalas, maior o nível de preconceito.

Como procedimento para a obtenção de autorização para a colheita de dados foi contactada a direção da IES, requerendo autorização para a recolha de dados na respetiva instituição. Após a obtenção da autorização, procedeu-se à recolha de dados, através da aplicação de Google Forms, com um questionário online, com pedido inicial do consentimento informado e foi facultado um e-mail para esclarecimento de dúvidas.

Foi garantido o tratamento ético dos participantes, respeitando a Declaração de Helsínquia, nomeadamente, em relação no que se refere à Proteção de dados dos participantes, tendo-se tratado a variável idade numa escala ordinal, por grupos etários, para garantir a confidencialidade das respostas, conforme indicações do parecer da Comissão de Ética favorável (Parecer 023/2018). Todos os estudantes optaram livremente por responder ao nosso questionário por intermédio da respetiva instituição de ensino.

Relativamente à análise dos dados foi utilizado o programa SPSS $® 21$ (Statistical Package for the Social Sciences), utilizando-se estatística descritiva e análise de correlações de Spearman entre as variáveis em estudo.

\section{RESULTADOS}

Inicialmente recolheram-se respostas de 100 participantes, contudo, excluiu-se um participante por não conclusão do preenchimento do questionário. Dos 99 participantes, 82,8\% identificam-se como sendo do género feminino, $16,2 \%$ do masculino e $1 \%$ de ambos os géneros. Da totalidade da amostra, $37,4 \%$ é do primeiro ano, $27,3 \%$ do segundo ano, $16,2 \%$ do terceiro ano e $19,2 \%$ do quarto ano. A maioria dos participantes do nosso estudo apresentam nacionalidade portuguesa (98\%), sendo $2 \%$ de naturalidade estrangeira (com nacionalidade Portuguesa), com idades compreendidas entre 17 e 19 anos (37,4\%), 20 a 22 anos $(35,4 \%), 23$ a 25 anos $(13,1 \%)$ e maiores de 25 anos $(14,1 \%)$. Dos estudantes, $86,9 \%$ é heterossexual, $7,1 \%$ são homossexuais, $4 \%$ são bissexuais e $2 \%$ prefere não responder. Relativamente ao local de residência, o meio rural corresponde a $39,4 \%$ dos participantes e o meio urbano corresponde a 60,6\%. Quanto à orientação religiosa, cristianismo $82,8 \%$, ateísmo $6,1 \%$, e outras $11,1 \%$. Quanto à orientação política, $70,7 \%$ não se identifica com nenhuma, 16,2\% com ideologia de direita, 10,1\% com ideologia de esquerda e $3 \%$ de centro. Por fim, no 


\section{Estudantes de enfermagem relativamente à comunidade LGBT: conhecimentos, atitudes e competência cultural}

questionário sociodemográfico à questão se tinham algum amigo que se identifique como LGBT, $60,6 \%$ dos participantes responderam "sim" e $39,4 \%$ dos participantes responderam "não".

Quanto à avaliação das diferenças de preconceito tendo em consideração os diferentes grupos de idade, verificou-se que só se encontram diferenças estaticamente significativas relativamente a F5Valorização Gay, não se verificando quanto aos fatores $F 1, r s=0,20 ; p=0,843$, e F4, $r s=-0,034 ; p=0,739$.

\section{Tabela 1}

Diferenças entre totais da subescala em função da idade

\section{Idade}

\begin{tabular}{|c|c|c|c|}
\hline 17 a 19 anos & 20 a 22 anos & 23 a 25 anos & mais de 25 anos \\
\hline $\mathrm{N}=37$ & $\mathrm{~N}=35$ & $\mathrm{~N}=13$ & $\mathrm{~N}=14$ \\
\hline
\end{tabular}

$\begin{array}{lllllllllll}\text { F5 - Valorização Gay } & 11,49 & 2,98 & 13,17 & 2,44 & 12,85 & 2,44 & 13,14 & 4,62 & , 040 & 0,207\end{array}$

Através da tabela 1, é possível verificar uma diferença estatisticamente significativa entre a idade, relativamente ao $F 5$-valorização gay $(p<0,05)$, constatando-se que os estudantes de enfermagem com idade compreendida entre os 20 e 22 anos apresentam valores mais elevados de discriminação, avaliados por este fator, seguidos dos estudantes com idade superior a 25 anos, dos estudantes com idades compreendidas entre os 23 e 25 anos e por último,

Tabela 2

Diferenças entre o total da subescala em função do ano de licenciatura

Ano de licenciatura

\begin{tabular}{cccccccccccc}
\cline { 2 - 10 } & \multicolumn{2}{c}{$\begin{array}{c}\text { 10 ano } \\
\mathrm{N}=37\end{array}$} & \multicolumn{2}{c}{$\begin{array}{c}\text { 20 ano } \\
\mathrm{N}=27\end{array}$} & \multicolumn{2}{c}{ 30 ano } & \multicolumn{2}{c}{ 40 ano } \\
& $\mathrm{N}=16$ & \multicolumn{2}{c}{$\mathrm{N}=19$} & & \\
& $\mathrm{M}$ & $\mathrm{DP}$ & $\mathrm{M}$ & $\mathrm{DP}$ & $\mathrm{M}$ & $\mathrm{DP}$ & $\mathrm{M}$ & $\mathrm{DP}$ & $\mathrm{P}$. & $\mathrm{r}_{\mathrm{s}}$ \\
\hline $\begin{array}{c}\text { F5 - Valorização } \\
\text { Gay }\end{array}$ & 11,22 & 2,90 & 13,67 & 3,34 & 12,81 & 2,61 & 13,05 & 2,72 &, 029 & 0,0219 \\
& & & & & & & & & & \\
\hline
\end{tabular}




\section{Estudantes de enfermagem relativamente à comunidade LGBT: conhecimentos, atitudes e competência cultural}

Através da tabela 2 é possível verificar uma diferença estatisticamente significativa entre $\mathrm{o}$ ano de licenciatura, relativamente ao F5-valorização gay $(p<0,05)$, constatando-se que os estudantes de enfermagem que frequentam o segundo ano de licenciatura, apresentam valores mais elevados de discriminação, avaliados por este fator, seguidos dos estudantes do quarto ano, dos estudantes do terceiro ano e por último, com resultados mais baixos, os estudantes do primeiro ano.
Quanto à avaliação das diferenças de preconceito tendo em consideração o sexo, verificou-se que não se encontram diferenças estaticamente significativas em nenhum fator. A saber:F1, $H(2)=1,868 ; p=0,393, F 4$, $H(2)=2,373 ; p=0,305$, e F5, $H(2)=2,294 ; p=0,318$.

Quanto à avaliação das diferenças de preconceito tendo em consideração a orientação sexual, verificouse que só se encontram diferenças estaticamente significativas relativamente a $\mathrm{F} 5$, não se verificando quanto aos fatores $F 1, H(3)=7,140 ; p=0,068$, e $F 4$, $H(3)=3,869 ; p=0,276$.

Tabela 3

Diferenças entre o total da subescala em função da orientação sexual

\section{Orientação Sexual}

\begin{tabular}{|c|c|c|c|c|c|c|c|c|c|c|}
\hline & \multicolumn{2}{|c|}{ Homossexual } & \multicolumn{2}{|c|}{ Heterossexual } & \multicolumn{2}{|c|}{ Bissexual } & \multicolumn{2}{|c|}{$\begin{array}{l}\text { Prefiro não } \\
\text { responder }\end{array}$} & \multirow[t]{2}{*}{ P. } & \multirow[t]{2}{*}{$\mathrm{H}$} \\
\hline & G.L. & Média & G.L. & Média & G.L. & Média & G.L. & Média & & \\
\hline F5 - Valorização & 7 & 9.1429 & 86 & 12,7907 & 4 & 11,5000 & 2 & 13,4949 & ,025 & 9,389 \\
\hline Gay & & & & & & & & & & \\
\hline
\end{tabular}

Através da tabela 3 é possível verificar uma diferença estatisticamente significativa entre as diferentes orientações sexuais relativamente ao F5-valorização gay $(p<0,05)$. Assim, constata-se que os estudantes de enfermagem que preferem não responder apresentam valores mais elevados de discriminação, avaliados por este fator, seguidos da orientação heterossexual, da orientação bissexual e, por último, com resultados mais baixos, os com orientação homossexual.

Quanto à avaliação das diferenças de preconceito tendo em consideração o local de residência, verificouse que só se encontram diferenças estaticamente significativas relativamente a $\mathrm{F} 5$, não se verificando quanto aos fatores $F 1, H(1)=0,240 ; p=0,624$, e $F 4$, $H(1)=1,701 ; p=0,192$. 


\section{Estudantes de enfermagem relativamente à comunidade LGBT: conhecimentos, atitudes e competência cultural}

Tabela 4

Diferenças entre total de subescala em função com o local de residência

Local de Residência

\begin{tabular}{|c|c|c|c|c|c|c|}
\hline & \multicolumn{2}{|r|}{ Rural } & \multicolumn{2}{|r|}{ Urbano } & \multirow[t]{2}{*}{ P. } & \multirow[t]{2}{*}{$\mathrm{H}$} \\
\hline & G.L. & Média & G.L. & Média & & \\
\hline F5 - Valorização Gay & 39 & 13,2051 & 60 & 12,0333 & 0,028 & 4,812 \\
\hline
\end{tabular}

Através da tabela 4 é possível verificar uma diferença estatisticamente significativa entre os diferentes locais de residência relativamente ao F5-valorização gay $(p<0,05)$. Assim, constata-se que os estudantes de enfermagem que residem em locais rurais apresentam valores mais elevados de discriminação, avaliados por este fator, seguidos do local urbano com resultados mais baixos.

Quanto à avaliação das diferenças de preconceito tendo em consideração a orientação religiosa, verificou-se que não se encontram diferenças estaticamente significativas em nenhum fator. Assim, constatou-se que $F 1, \quad H(2)=0,445, p=0,801 ; \quad F 4$, $H(2)=4,334, p=0,114$; e F5, $H(2)=2,433, p=0,296$.

Quanto à avaliação das diferenças de preconceito tendo em consideração a orientação política, verificouse que só se encontram diferenças estaticamente significativas relativamente a $F 1$, não se verificando quanto aos fatores $F 4, H(3)=1,465 ; p=0,690$, e $F 5$, $H(3)=6,884 ; p=0,076$.

Tabela 5

Diferenças entre total de subescala em função com a orientação política

Orientação Política

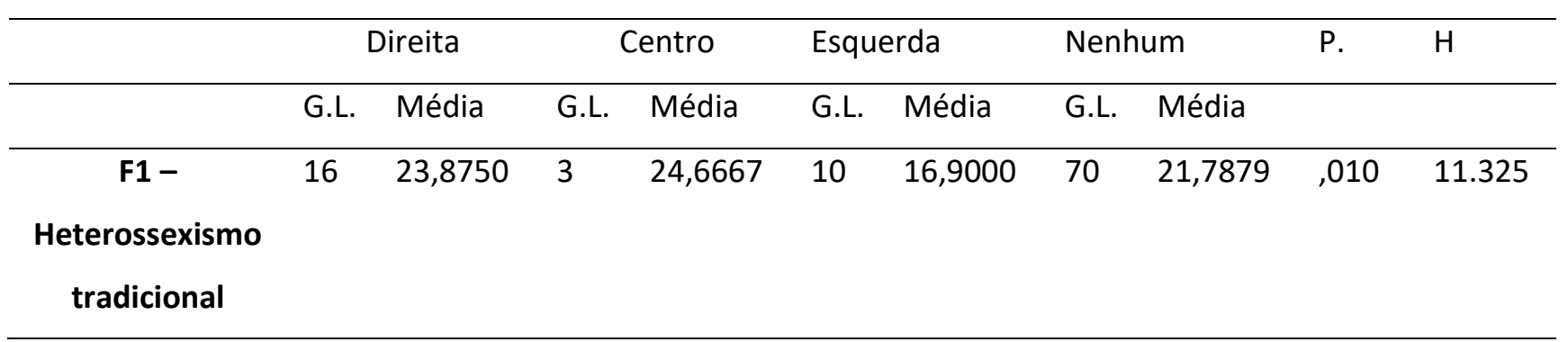

Através da tabela 5 é possível verificar uma diferença estatisticamente significativa entre as diferentes orientações políticas relativamente ao F1heterossexismo tradicional $(p<0,01)$. Assim, constatase que os estudantes de enfermagem que apresentam uma orientação de centro são os que apresentam valores mais elevados de discriminação, avaliados por este fator, seguidos da orientação de direita, dos que afirmam não ter orientação política e, por último, com resultados mais baixos, os com orientação de esquerda. 


\section{Estudantes de enfermagem relativamente à comunidade LGBT: conhecimentos, atitudes e competência cultural}

Quanto à avaliação das diferenças de preconceito tendo em consideração a convivência frequente com amigos/familiares que se identifiquem como LGBT, verificou-se que só se encontram diferenças

Tabela 6

Diferenças entre total de subescala em função com a familiaridade com a comunidade LGBT

Ter ou não ter amigos LGBT

\begin{tabular}{|c|c|c|c|c|c|c|}
\hline & \multicolumn{2}{|c|}{ Ter amigos } & \multicolumn{2}{|c|}{ Não ter amigos } & \multirow[t]{2}{*}{$p$} & \multirow[t]{2}{*}{$\mathrm{H}$} \\
\hline & G.L. & Média & G.L. & Média & & \\
\hline F1 - & 60 & 12,1167 & 39 & 13,0769 & ,000 & 16,093 \\
\hline \multicolumn{7}{|c|}{ Heterossexismo } \\
\hline tradicio & & & & & & \\
\hline
\end{tabular}

Através da tabela 6 é possível verificar uma diferença estatisticamente significativa entre os que convivem frequentemente com amigos/familiares que se identifiquem como LGBT, relativamente ao F1heterossexismo tradicional $(p<0,01)$. Assim, constatase que os estudantes de enfermagem que não convivem frequente com amigos/familiares que se identifiquem como LGBT apresentam valores mais elevados de discriminação, avaliados por este fator, do

Tabela 7

Correlação das subescalas em função dos conhecimentos sobre LGBT

Nível de Conhecimentos

\begin{tabular}{ccc} 
Nível de Conhecimentos & & \\
\cline { 3 - 3 } & \multicolumn{1}{c}{ Sig } & $\mathrm{r}_{\mathrm{s}}$ \\
\hline F1 - Heterossexismo Tradicional &, 010 &,- 258 \\
\hline F4 - Negação da discriminação homofóbica &, 004 &,- 289 \\
\hline F5 - Valorização Gay & & \\
\hline
\end{tabular}

que estudantes de enfermagem que apresentam uma convivência frequente.

Quanto à avaliação das diferenças de preconceito tendo em consideração o nível de conhecimento, verificou-se que só se encontram diferenças estaticamente significativas relativamente a F4 e F5, não se verificando quanto aos fatores $F 1, r s=-0,258$; $p=0,010$. 


\section{Estudantes de enfermagem relativamente à comunidade LGBT: conhecimentos, atitudes e competência cultural}

Através da tabela 7 é possível verificar uma correlação negativa estatisticamente significativa entre os a variável conhecimentos e os fatores F4-negação da discriminação homofóbica e F5- valorização gay $(p<0,01)$.

\section{DISCUSSÃO}

A amostra recolhida, apesar de ter sido uma amostra representativa da IES (por incluir a quase totalidade dos estudantes de licenciatura) é limitada no que se refere à representabilidade da população de estudantes de enfermagem portugueses, apesar das caraterísticas que apresenta, como a maior proporção do género feminino, ser comum à generalidade da população objetivada, pelo que pode ser afirmado como um estudo exploratório relevante para o conhecimento desta temática.

Segundo Unlu e colaboradores (2016) e Pinto (2011) seria espectável uma razão diretamente proporcional entre valores discriminatórios e a idade, contudo não foi consistente com os resultados obtidos, pois os dados obtidos, apesar de revelarem diferenças estatisticamente significativas, não são num sentido completamente linear, pois o grupo etário que apresentou valores mais elevados de discriminação foi o dos 20 aos 22 anos, seguido do com mais de 25 anos, e o com menores valores de discriminação, de Valorização Gay, o grupo mais jovem. Geralmente, a idade varia tendencialmente com o ano académico (Pinto, 2011) e, os dados obtidos indicam o segundo ano como um ano com valores discriminatórios mais elevados, seguido do quarto ano, terceiro e primeiro ano, evidenciando diferenças estatisticamente significativas relativamente à Valorização Gay, ainda que não linear com o avançar dos anos de formação académica. Apesar de não se encontrar um sentido consistente das diferenças encontradas, os resultados corroboram a literatura, com as variáveis idade e ano académico a demonstrarem ser relevantes nas atitudes e competência cultural dos estudantes de enfermagem relativamente à comunidade LGBT. Possivelmente, se a variável idade tivesse sido possível de recolher como variável contínua e não em grupos etários, esta associação poderia ter uma leitura com melhores possibilidades de análise. A dimensão da IES em que foi realizado o estudo poderia permitir a identificação dos participantes se a recolha da variável fosse realizada numa escala contínua e não por grupos etários, pelo que esta variável deve ser estudada dessa forma em amostras mais alargadas, já que apresenta uma associação significativa que importa clarificar. Unlu, et al. (2016) demonstrou que os estudantes de enfermagem do género masculino apresentam atitudes mais negativas perante Lésbicas e Gays do que estudantes de enfermagem do género feminino, o que não foi corroborado por este estudo, pois não se encontraram diferenças significativas entre género.

No parâmetro sociodemográfico de orientação sexual, os resultados obtidos corroboram a literatura encontrada, demonstrando diferenças significativas, com valores mais elevados de discriminação dos Heterossexuais, avaliados pelo factor de Valorização Gay, seguidos dos Bissexuais e com os menores valores dos Homossexuais.

Os resultados relativamente ao local de residência em meio rural apontam para um nível de discriminação mais elevado dos que os residentes em meio urbano, corroborando os dados obtidos de Pinto (2011) e contrariando os dados apresentados por Unlu e colaboradores (2016). 


\section{Estudantes de enfermagem relativamente à comunidade LGBT: conhecimentos, atitudes e competência cultural}

No parâmetro sociodemográfico de orientação religiosa, ao contrário da maioria da literatura, que encontrou evidência de que existem diferenças ao nível de discriminação (Richardson, Ondracek, \& Anderson, 2016; Rowniak, 2015), não se encontraram diferenças de preconceito significativas, à semelhança de outros estudos, como o Cornelius e Carrick (2015). Relativamente à orientação política verificou-se que os estudantes de enfermagem com orientação de centro apresentam um maior nível discriminatório seguido da orientação de direita, o que condiz com os resultados de estudos anteriores, como os referidos por Pinto (2011).

No parâmetro sociodemográfico de familiaridade com a comunidade LGBT, verificou-se que os estudantes de enfermagem que contactam frequentemente com pessoas pertencentes a esta comunidade apresentam níveis de discriminação mais reduzidos. Deste modo, os dados obtidos neste estudo espelham os resultados obtidos por outros estudos como Unlu e colaboradores (2016) e Pinto (2011) e, também, Kelly e colaboradores (2008).

O variável nível de conhecimento apresentam correlação inversa com as subescalas de negação da discriminação homofóbica e da valorização gay, encontrando-se que quanto maior o nível de conhecimentos dos estudantes de enfermagem menor os níveis de discriminação, conforme também foi verificado por Cornelius et Carrick (2015).

Estudos atuais defendem a importância de diminuição desta lacuna nos cursos de Enfermagem a nível global, com vista a melhorar o conhecimento cultural e as competências dos enfermeiros para melhorar os resultados dos cuidados de saúde nesta população vulnerável (Maley \& Gross, 2019; Morris et al., 2019;
Guzman et al., 2018). Alguns destes estudos concluíram acerca das estratégias que se revelaram eficazes, nomeadamente as que promovem 0 conhecimento das necessidades de cuidados de saúde de pessoas LGBTQ e que promovem o conforto ao trabalhar com pacientes LGBTQ (Morris et al., 2019), bem como, a realização de redações reflexivas sobre as disparidades de saúde nas populações LGBT (Maley \& Gross, 2019).

Ao longo deste estudo verificamos a existência de limitações, sendo elas, o número reduzido da amostra, a maioria ser do sexo feminino, as idades distribuídas entre os diferentes anos de licenciatura serem muito variados e a amostra estar restrita a uma IES. Sugerimos, assim, a continuidade de estudos de investigação futura nesta área, com inclusão de amostras alargadas, com outras IES, com a possibilidade de recolha da variável idade de forma contínua, para poder retirar conclusões mais consistentes e com maior potencial de generalização, para além de investigação que monitorize os efeitos de formação adequada sobre esta temática, na atitude e competências dos estudantes de enfermagem, de outras áreas da saúde, profissionais e da comunidade em geral.

\section{CONCLUSÃO}

Com este estudo verificou-se que fatores como a idade e o ano letivo, a orientação sexual, o local de residência, a orientação política e a familiaridade com pessoas LGBT e o nível de conhecimentos têm interferência na atitude e competência cultural dos estudantes de enfermagem relativamente à comunidade LGBT. Os resultados deste estudo exploratório, apesar de ser limitado a uma IES, 


\section{Estudantes de enfermagem relativamente à comunidade LGBT: conhecimentos, atitudes e \\ competência cultural}

corroboram conclusões da literatura emergente sobre esta temática. Apesar de não se encontrar uma leitura linear da interferência da idade e do ano letivo, confirma-se a relevância desta variável na atitude e competência cultural dos estudantes nesta área. A orientação sexual heterossexual, o local de residência rural, a orientação política de centro e o não ter contacto com pessoas LGBT, demonstram ser fatores de risco para uma atitude discriminatória. Um nível de conhecimentos mais elevado revela estar associado a uma atitude menos discriminatória, propiciadora de melhor competência cultural.

Estes resultados fornecem implicações com indicação de reforço da sensibilização e formação sobre esta temática a nível académico, com especial atenção a públicos-alvo que apresentam maior risco de atitudes discriminatórias, nomeadamente com orientação heterossexual, com residência em meios rurais, com orientação política de centro e os que não têm contacto com pessoas LGBT. Considera-se urgente a inclusão deste tema nas unidades curriculares, com seminários e ações de sensibilização para a não discriminação de pessoas LGBT, o que pode ser fundamental para a preparação enquanto profissionais de saúde, mas também pelo seu papel de educadores em saúde na comunidade.

\section{REFERÊNCIAS BIBLIOGRÁFICAS}

American Psychiatric Association . (2014). Manual de Diagnóstico e Estatística das Perturbações Mentais: DSM-5. Lisboa: Artmed.

Assembleia da República. (31 de maio de 2010). Lei n.o 9/2010 de 31 de Maio . Diário da República, p. 1853.

Betancourt, D. A. (2015). Madeleine Leininger and the Transcultural Theory of Nursing. The Downtown Review, 2, 1-8.
Campbell, R. J. (2009). Dicionário de Psiquiatria. Porto: Artmed Editora.

Cornelius, J. B., \& Carrick, J. (2015). A Survey of Nursing Students' Knowledge of and Attitudes Toward LGBT Health Care Concerns. (B. J. Patterson, Ed.) Nursing Education Perspectives, 36(3), 176-178.

Davidson, M. R. (2014). Development and validation of the transgender prejudice scale (Tese de Mestrado apresentada à Western Washington University). Washington.

Guzman, F. L., Moukoulou, L. N. N., Scott, L. D. \& Zerwic, J. J. (2018). LGBT inclusivity in health assessment textbooks. Journal of Professional Nursing, 34(6):483-487. doi: 10.1016/j.profnurs.2018.03.001

International Lesbian and Gay Association - ILGA. (2019). Igla-Portugal. Fonte: Direitos LGBTI em destaque na celebração dos 45 anos do 25 de Abril. Retirado de https://ilga-portugal.pt/noticias/1097.php

Lim, F. A., \& Hsu, R. (2016). Nursing Students' Attitudes Toward Lesbian, Gay, Bisexual, and Transgender Persons: An Integrative Review. Nursing Education Perspectives, 37, 144-152.

Lopes, D., Oliveira, J. M., Nogueira, C., \& Grave, R. (27 de abril de 2016). The Social Determinants of Polymorphous Prejudice Against Lesbian and Gay Individuals: the Case of Portugal. Sex Res Soc Policy, 5670.

Maley, B. \& Gross, R. (2019). A writing assignment to address gaps in the nursing curriculum regarding health issues of LGBT+ populations. Nursing Forum, 54(2):198-204. DOI: 10.1111/nuf.12315

Morris, M., Cooper, R. L., Ramesh, A., Tabatabai, M., Arcury, T. A., Shinn, M., ... Matthews-Juarez, P.(2019). Training to reduce LGBTQ-related bias among medical, nursing, and dental students and providers: a systematic review. BMC Medical Education,19: 325. doi: 10.1186/s12909-019-1727-3

Orgel, H. (2017). Improving LGBT Cultural Competence in Nursing Students: An Integrative Review. The ABNF Journal, 14-18.

Pinto, D. P. (2011). Discriminação, preconceito $e$ atitudes relativamente a lésbicas: Estudo numa amostra de estudantes de enfermagem (Tese de Mestrado apresentada à Universidade do Minho). Minho.

Richardson, B. P., Ondracek, A. E., \& Anderson, D. (2016). Do student nurses feel a lack of comfort in 
providing support for Lesbian, Gay, Bisexual or Questioning adolescents: what factors influence their comfort level? (J. W. Sons, Ed.) Journal of Advanced Nursing, 1196-207.

Rodrigues, F. R., Martins, J. J., \& Pereira, M. L. (2013). Competência cultural: an lise do conceito segundo a metodologia tradicional de Walker e Avant. E-REI: Revista de Estudos Internacionais do CEI, 1-10.

Rowniak, S. (2015). Factors Related to Homophobia Among Nursing Students. Journal of Homosexuality, 1228-1240.
Strong, K. L. (2013). Assessing Undergraduate Nursing Students' Knowledge, Attitudes and Cultural Competence in Caring for LGBT Patients. Honors Projects.

UNESCO (2016). Out in the Open: Education Sector Responses to Violence Based on Sexual Orientation and Gender Identity/Expression. Paris: UNESCO.

Unlu, H., Beduk, T., \& Duyan, V. (2016). The attitudes of the undergraduate nursing students towards lesbian women and gay men. Journal of Clinical Nursing, 36973706. 